\title{
RELACIÓN ENTRE EL APRENDIZAJE DE LA VOZ ERIGMOFÓNICA $Y$ LOS NIVELES DE ANSIEDAD Y DE DEPRESIÓN EN PACIENTES LARINGECTOMIZADOS TOTALES
}

\author{
RELATIONSHIP BETWEEN THE ESOPHAGEAL VOICE LEARNING AND ANXIETY AND \\ DEPRESSION LEVELS IN TOTAL LARYNGECTOMEES
}

\author{
Eduardo Zubiri', María Milagros Pollán², $M^{a}$ Inés Gabari² \\ ' Centro de Psicología del Lenguaje y Psicoterapia "ETHOS". Pamplona. Asociación Navarra de Laringectomizados. \\ 2 Universidad Pública de Navarra.
}

Resumen

La pérdida de la voz laríngea es la principal consecuencia que sufren los laringectomizados totales, por lo que el aprendizaje de una nueva voz es el objetivo principal de la rehabilitación de estos pacientes. La voz erigmofónica es una de las posibilidades que tiene estos pacientes para recuperar la capacidad de comunicación oral de una manera natural.

La laringectomía total se asocia a consecuencias psicológicas, como la ansiedad y la depresión, frecuentemente presentes en la enfermedad oncológica.

En la investigación que presentamos, se hace referencia a la posible incidencia de algunas de las características de la voz erigmofónica sobre los niveles de ansiedad y de depresión de estos pacientes. Los cambios observados en estas respuestas en función de la percepción que tienen sobre su nueva voz, de los cambios experimentados en su aprendizaje, de la importancia que ha supuesto para ellos y si tienen impresión de habla correcta con las personas con las que se comunican. Se ha valorado la incidencia que ha supuesto el tiempo que les ha costado alcanzar un nivel mínimo de dominio de la voz erigmofónica necesaria para mantener comunicación oral.

Los resultados obtenidos confirman la significación o valor predictivo de tres de las variables estudiadas: Las personas que perciben
Abstract

The loss of laryngeal voice is the main consequence suffered by total laryngectomy, so learning a new voice is the main objective of the rehabilitation of these patients. Esophageal voice is one of the possibilities for patients to recover oral communication skills in a natural way.

Total laryngectomy is associated with psychological consequences such as anxiety and depression often present in cancer disease. The present research is about the potential impact of some of the characteristics of the esophageal voice on levels of anxiety and depression in these patients. The observed changes in these responses in terms of perceptions about his new voice, the changes in their learning, the importance that has meant for them and if they have impression of correct speech and with whom they communicate. We evaluated the impact of time it took them to reach a minimum level of mastery of esophageal voice necessary to maintain oral communication.

The results obtained confirm the significance or predictive value of three of the variables studied: People who perceive they have learned to speak, they speak well and speak with anyone (not just family) are less depressed. In the case of anxiety are no effects.

\section{Correspondencia:}

Eduardo Zubiri Vidaurreta Centro de Psicología del Lenguaje y Psicoterapia Psicoanalítica"

C/ Iturralde y Suit, 1-6 Dcha.

31004 Pamplona (Navarra)

E-mail: ezubiri@ethospsicologos.com 
que han aprendido a hablar, que hablan bien y que hablan con cualquiera (no solo con familiares) están menos deprimidos. En el caso de la ansiedad no se encuentran efectos.

Hemos elaborado un perfil del paciente que no disminuye su nivel de depresión después de haber adquirido la voz erigmofónica: considera que ha aprendido a hablar con dificultad, ya que le ha costado más de 6 meses; pero no se siente nada satisfecho con su nueva voz, tiene la impresión de no hablar correctamente, habla solamente con familiares y la pérdida de la voz le supuso un sentimiento de incapacidad. La laringectomía le afectó mucho psicológicamente, familiarmente, socialmente y laboralmente (está en paro). Se siente muy afectado físicamente, con molestias por el traqueostoma, dificultades en la respiración y dificultades para tragar alimentos. Es relativamente joven (54 años) y está viudo.

Palabras clave: Laringectomía total, voz erigmofónica, ansiedad, depresión.
We have developed a patient profile that does not diminish their level of depression after having acquired the voice erigmofónica: Consider who has learned to speak with difficulty, and that has cost more than 6 months, but does not feel at all satisfied with their new voice, has the impression of not speaking properly, speak only with family and lost his voice was a sense of incapacity. Laryngectomy psychologically affected him deeply, familiarly, socially and occupationally (is unemployed). He feels physically affected with discomfort for the tracheostoma, difficulty in breathing and swallowing difficulties. The patient is relatively young (54 years) and is a widower.

Keywords: Total laryngectomy, esophageal voice, anxiety, depression.

\section{INTRODUCCIÓN}

La laringectomía total supone un importante impacto en quienes la padecen, debido a las consecuencias que la intervención y la enfermedad producen en ellos, no sólo en la imagen corporal y la comunicación, sino también en aspectos emocionales como el incremento de ansiedad y depresión. Estos niveles de ansiedad y depresión se asocian, en este caso, a la pérdida de la voz y a lo que esto supone: pérdida de la identidad, temor a la recaída, situación de indefensión y de incertidumbre ante la comunicación interpersonal, etc. que llevan asociados distintos síntomas, como la irritabilidad, alteraciones psicomotoras, alteraciones del pensamiento y de la propia autoestima. Alteraciones que no se producen en aquellos pacientes que no han perdido su voz tras la operación (laringectomía parcial), al menos con la misma intensidad, como indica González ${ }^{(1)}$.

Son múltiples los estudios referidos a las consecuencias psicológicas de la laringectomía total, generalmente referidos a la ansiedad y a la depresión, relacionándolos con la pérdida de la voz laríngea; pero no hemos encontrado estudios que se refieran al estado emocional de los pacientes después de haber adquirido la voz erigmofónica y qué influencia puede tener ésta en su estado emocional.

En este artículo pretendemos comprobar si existe alguna relación entre el aprendizaje de la voz erigmofónica y los estados de ansiedad y depresión que se producen en los pacientes laringectomizados totales; para los que como dice $\mathrm{Algaba}^{(2)}$, las dos primeras consecuencias directas de la laringectomía son la privación de la voz natural, la voz laríngea, y la necesidad de respirar a través de un traqueostoma, En 
este caso nos interesa de manera especial la pérdida de la voz laríngea y su sustitución por una nueva voz que los pacientes deben aprender y a la se tiene que adaptar y con la que se tienen que identificar: La voz erigmofónica o esofágica.

\section{Voz erigmofónica o esofágica}

La voz erigmofónica (del griego erigmos: eructar) o voz esofágica es el sistema más natural de expresión oral para el paciente laringectomizado, y constituye el primer objetivo que nos marcamos en el proceso rehabilitador.

Las condiciones físicas y psicológicas que requiere el aprendizaje de la voz erigmofónica hacen que no todos los pacientes sean capaces de lograrla, ni de alcanzar el mismo grado de satisfacción con la misma los que lo consiguen, por lo que no tenemos que descartar la posibilidad del acceso a la comunicación oral a través de otros métodos como la voz traqueoesofágica y laringe artificial o electrolaringe (García y O'Connor $^{(3)}$ ).

La voz erigmofónica va a tener unas limitaciones claras respecto a la voz laríngea:

- La intensidad de la voz va a ser menor y ello va a suponer que el laringectomizado tenga dificultades para comunicarse en ambientes ruidosos o para hablar en público.

- También va a ser menor la duración de la emisión sonora. Ello es debido a la menor capacidad de la cavidad esofágica; no obstante, con la práctica, se logra desarrollar el tamaño y el tono muscular consiguiendo aumentar tanto el volumen como la duración de la emisión sonora.

- El timbre de la voz es otro de los aspectos que va a variar respecto de la voz laríngea. Depende de la vibración de la pseudoglotis al paso del aire y de la resonancia que tenga la cavidad faríngea y la bucal, es decir, de su amplitud, de sus movimientos y de la ausencia de secreciones en estas zonas. El sonido emitido por el esófago y la pseudoglotis, al pasar por el tubo faringo-bucal se va a ir modulando según la forma que tenga, adquiriendo mayor resonancia o ensordeciéndose, según la posición en que se coloquen la lengua y el velo del paladar en estas cavidades. Será importante, por tanto, relajar la faringe para ampliarla y producir la voz con el mínimo grado de tensión muscular del cuello.

- La fluidez va a ser otro de los factores diferenciadores respecto de la voz laríngea, ya que conseguir una emisión de voz sin interrupciones ni bloqueos no está al alcance de todos. Podríamos decir que la longitud media de la frase disminuye claramente; aunque hay algunos pacientes que adquieren un nivel de habla próximo al normal.

- Por último, otro factor diferenciador va a ser la capacidad de entonación. La entonación ascendente de la prosodia (interrogaciones, exclamaciones...) se realiza aumentando la cantidad de aire emitido, la frecuencia de vibración de la pseudoglotis y la amplitud de la faringe. Para ello se requiere que la cavidad esofágica y la pseudoglotis estén bastante desarrolladas y que la faringe tenga un buen grado de flexibilidad para poder subir y bajar sincrónicamente con la lengua. Esto sólo está al alcance de algunos, ya que las condiciones postoperatorias varían mucho de unos a otros.

Todos estos factores hacen que la voz erigmofónica tenga unas características diferentes a la voz laríngea y el paciente ha de aceptarlas y aprender a identificarse con ellas; de tal manera que, como dicen Borragán, González y Agudo(4): "una laringectomía (...) no debería vivirse como una pérdida, sino como una adaptación a una 
nueva situación, una nueva oportunidad para seguir viviendo" (pág. 50).

\section{Aprendizaje de la voz esofágica}

La voz erigmofónica o voz esofágica, recibe el nombre de la utilización del aire de la primera porción del esófago superior, inmediatamente por debajo del anillo o esfínter esofágico, formado por el músculo cricofaríngeo.

Este aire, previamente deglutido o introducido en esta porción del esófago, es eructado o soplado hacia la faringe y hacia la cavidad oral, produciendo un sonido (eructo), que será articulado por los órganos articulatorios de igual forma que lo era el soplo pulmonar tras pasar por la glotis.

El soplo esofágico es totalmente independiente del soplo traqueal o pulmonar, que ahora, tras la laringectomía, desemboca en el cuello por el estoma. Este nuevo vibrador recibe el nombre de neoglotis o pseudoglotis.

Recordando las tres condiciones necesarias para la producción del habla, en este caso:

- La fuente de aire la constituye el esófago.

- La estructura estrecha, flexible y elástica con capacidad para vibrar, la constituye la neoglotis.

- Las cavidades de resonancia son las mismas que antes de la laringectomía.

En definitiva, en principio se dan las condiciones mínimas para que el laringectomizado sea capaz de producir voz; otra cosa será la dificultad o facilidad que en realidad se encuentre en cada caso en particular.

La mayor dificultad de este nuevo proceso, suele radicar en la introducción del aire en esa porción alta del esófago, traspasando el esfínter esofágico.
El objetivo del presente trabajo es exponer los datos descriptivos de aprendizaje de la voz erigmofónica de pacientes totalmente laringectomizados en cuanto a las siguientes variables: Tiempo de aprendizaje, ¿Ha aprendido a hablar?, ¿Cómo habla? Impresión de habla correcta, Satisfacción, con su nueva voz ¿Con quién habla? para a continuación valorar su efecto en los niveles de ansiedad y depresión.

\section{MÉTODO}

La muestra esta compuesta por 40 sujetos laringectomizados totales, 39 hombres y una mujer, 34 de los cuales pertenecen a la Asociación Navarra de Laringectomizados y 6l a Escuela de Patología del Lenguaje del Hospital de San Pablo y de la Santa Cruz de Barcelona, con edades comprendidas entre 44 y 89 años, siendo la edad media de 61,35 años, la mediana 62 años y una desviación típica 8,54.

Se trata de un estudio de tipo observacional descriptivo que se ha realizado en dos fases: pre test y pos test. En la primera fase se han analizado los niveles de ansiedad y de depresión de los pacientes laringectomizados antes de comenzar el aprendizaje de la voz erigmofónica; para lo que hemos utilizado La Escala Hospitalaria de Ansiedad y Depresión (HAD) de Zigmond y Snaith ${ }^{(5)}$, con adaptación española de Caro e Ibáñez (1992) ${ }^{(6)}$ y el Cuestionario de Ansiedad-Estado (STAI-E) de Spielberger ${ }^{(7)}$. El HAD se considera que es más eficaz en la medición de la depresión que en la de la ansiedad, por lo que hemos reforzado la medición de los niveles de ansiedad con la aplicación del STAI, que mide ansiedad-rasgo y ansiedad-estado. En la $2^{\circ}$ fase, que corresponde al momento en el que los pacientes han conseguido un nivel de aprendizaje de la voz erigmofónica que les permite mantener una conversación básica. Además de la utilización de los dos cuestio- 
narios ya señalados para comprobar las variaciones en los niveles de ansiedad y de depresión, se ha aplicado un cuestionario general, elaborado para la ocasión, en el que, además de otros datos de tipo biográfico y relativos a los diversos grados de afectación (psicológica, familiar, social, etc.) y de dificultad para diversas funciones, se les ha preguntado por aspectos que tiene que ver con la calidad, la satisfacción, la utilidad y uso de la voz erigmofónica.

\section{RESULTADOS}

Después del periodo de aprendizaje de la voz erigmofónica, en el momento del retest se ha preguntado a los pacientes laringectomizados sobre los siguientes aspectos de su habla: tiempo de aprendizaje, si ha aprendido a hablar, impresión de habla correcta, cómo hablan, con quién, si dirigen la palabra a desconocidos y si se sienten satisfechos con su nueva voz, que constituyen las variables independientes.

\section{Variables independientes}

Tiempo de aprendizaje

El $90 \%$ de los pacientes ha adquirido un nivel de aprendizaje de la voz erigmofónica, que le permite mantener una conversación en situación favorable, es decir sin ruido ambiental, entre 0 y 12 meses. Solamente un $10 \%$ ha necesitado más de un año para pasar al nivel II. Pero es relevante que el $42,5 \%$ lo haga entre 0 y 3 meses y que otro $30 \%$ lo haga entre 4 y 6 meses, lo cual supone que casi 3 de cada 4 pacientes consiga un nivel de voz erigmofónica funcional en medio año (recordamos que reciben solamente dos horas semanales de instrucción). Este porcentaje sube al 85\% a los 9 meses (ver tabla 1).
Tabla 1. Tiempo de aprendizaje.

\begin{tabular}{|r|l|r|}
\hline Frecuencia & \multicolumn{1}{|c|}{$\begin{array}{c}\text { Intervalo } \\
\text { meses }\end{array}$} & \multicolumn{1}{c|}{ Porcentaje } \\
\hline 17 & $0-3$ & 42,5 \\
\hline 12 & $4-6$ & 30,0 \\
\hline 5 & $7-9$ & 12,5 \\
\hline 2 & $9-12$ & 5,0 \\
\hline 4 & $>12$ & 10,0 \\
\hline 40 & Total & 100,0 \\
\hline
\end{tabular}

¿Ha aprendido a hablar?

En el momento de realizar el retest, el $90 \%$ de los pacientes considera que ha aprendido a hablar y hay un $10 \%$ que considera que no ha aprendido a hablar. Este dato nos indica que el criterio del terapeuta, que considera que el paciente ya ha logrado un nivel suficiente para pasar al nivel II de aprendizaje, puede no coincidir con la apreciación del propio paciente, probablemente por la expectativa de éste sobre la calidad de su voz, su funcionalidad y sus límites y, aunque es capaz de hablar, no considera que su voz sea adecuada (ver tabla 2).

\section{Tabla 2. ¿Ha aprendido a hablar?}

\begin{tabular}{|c|r|r|}
\hline $\begin{array}{c}\text { ¿Ha aprendido } \\
\text { a hablar? }\end{array}$ & Frecuencia & \multicolumn{1}{c|}{ Porcentaje } \\
\hline No & 4 & 10,0 \\
\hline $\mathrm{Si}$ & 36 & 90,0 \\
\hline Total & 40 & 100,0 \\
\hline
\end{tabular}

\section{¿Cómo habla?}

Solamente el $12,5 \%$ de los pacientes considera que habla bien; el 85\% considera que solamente habla regular. Esta apreciación sobre su nivel de habla nos está indicando las dificultades que tienen los pacientes para lograr un habla fluida, con 
suficiente volumen y funcional en cualquier situación y la valoración que hacen de su nueva voz. Los pacientes se hacen conscientes de las limitaciones que supone la voz erigmofónica respecto de la voz laríngea que poseían.

Es reseñable que ningún paciente considera que habla mal, a pesar de que el $10 \%$ de los pacientes consideran que no han aprendido a hablar; de ellos, un 25\% no contesta y el $75 \%$ consideran que hablan regular; por lo que de nuevo nos enfrentamos a la interpretación personal del concepto y para ellos hablar regular no es suficiente y consideran que no han aprendido a hablar (ver tabla 3 ).

Tabla 3. ¿Cómo habla?

\begin{tabular}{|c|r|r|}
\hline ¿Cómo habla? & Frecuencia & \multicolumn{1}{c|}{ Porcentaje } \\
\hline Bien & 5 & 12,5 \\
\hline Regular & 34 & 85,0 \\
\hline N/C & 1 & 2,5 \\
\hline Total & 40 & 100,0 \\
\hline
\end{tabular}

Impresión de habla correcta

A la pregunta referida a la impresión que tienen los pacientes sobre si su habla es correcta o no, los resultados indican que se dan ciertas similitudes con la anterior pregunta; así, una mayoría $(77,5 \%)$ considera que su habla no es correcta $y$ hay un grupo menor $(22,5 \%)$ para el que sí lo es (ver tabla 4).

\section{Tabla 4. Impresión de habla correcta.}

\begin{tabular}{|l|r|r|}
\hline $\begin{array}{c}\text { Impresión } \\
\text { habla correcta }\end{array}$ & \multicolumn{1}{|c|}{ Frecuencia } & \multicolumn{1}{c|}{ Porcentaje } \\
\hline $\mathrm{No}$ & 31 & 77,5 \\
\hline $\mathrm{Si}$ & 9 & 22,5 \\
\hline Total & 40 & 100,0 \\
\hline
\end{tabular}

\section{Satisfacción con su nueva voz}

Sorprende que a pesar de que la mayoría de los pacientes piensa que no habla bien o sólo regular y que su habla no es correcta, cuando les preguntamos sobre qué grado de satisfacción tienen con su nueva voz, el $65 \%$ de los pacientes refiere que se sienten bastante $(42,5 \%)$ o muy $(22,5 \% /)$ satisfechos y solamente el $20 \%$ se siente poco satisfecho con su nueva voz. Solamente el 2,5\% está nada satisfecho (tabla 5).

\section{Tabla 5. Satisfacción con su nueva voz.}

\begin{tabular}{|c|r|r|}
\hline $\begin{array}{c}\text { Satisfacción con } \\
\text { su nueva voz }\end{array}$ & Frecuencia & Porcentaje \\
\hline Nada & 1 & 2,5 \\
\hline Poco & 8 & 20,0 \\
\hline Bastante & 17 & 42,5 \\
\hline Mucho & 9 & 22,5 \\
\hline N/C & 5 & 12,5 \\
\hline Total & 40 & 100,0 \\
\hline
\end{tabular}

Estas aparentes incoherencias las podemos explicar por el conocimiento que tienen los propios pacientes de las limitaciones que tiene la voz erigmofónica $y$, al mismo tiempo se comparan con otros laringectomizados "veteranos" y comprueban el nivel que han adquirido y que, por tanto, también ellos podrían adquirir. Cuando dicen que están satisfechos de su nueva voz, no quieren decir que no les gustaría hablar mejor, con mayor fluidez, mayor volumen o mejores registros.

Desde el análisis individualizado de cada caso, observamos bastante coherencia con los resultados de las dos anteriores preguntas; es decir, casos que consideran que hablan bien y que tienen la impresión de que su habla es correcta, están muy satisfechos con su nueva voz y los casos 
que a pesar de considerar que su nivel de habla es regular, consideran que su habla es correcta y está bastante satisfecho con su nueva voz.

También se da la paradoja de que hay un grupo de pacientes numeroso $(40 \%)$, que no tienen la impresión de que su habla sea correcta, sin embargo están bastante o muy satisfechos con su nueva voz; por lo que podemos considerar que, de alguna manera, sus expectativas se ha visto satisfechas y han logrado una voz funcional que les permite manejarse y les ha dado cierta independencia en su vida cotidiana.

Por último, el 20\% que está poco satisfecho con su nueva voz, comprobamos también consideran que su nivel de habla es solamente regular y no tienen la impresión de que su habla sea correcta.

\section{¿Con quién habla?}

Pretendemos explorar si los pacientes que han logrado este primer nivel conversacional tienen alguna limitación en su actividad comunicativa; así comprobamos que el $75 \%$ dirige la palabra a cualquiera, por lo que podríamos deducir que no tienen ninguna limitación comunicativa; sin embargo, un 25\% limita su acción comunicativa al ámbito familiar próximo, esposa e hijos principalmente (ver tabla 6).

\section{Tabla 6. ¿Con quién habla?}

\begin{tabular}{|c|r|r|}
\hline ¿Con quién habla? & Frecuencia & Porcentaje \\
\hline Familiares & 10 & 25,0 \\
Con cualquiera & 30 & 75,0 \\
Total & 40 & 100,0 \\
\hline
\end{tabular}

En este apartado, al hacer el análisis individualizado volvemos a encontrarnos con datos que nos llaman la atención, ya que observamos que hay pacientes que están poco satisfechos con su nueva voz y que no tienen la impresión de habla co- rrecta y creen que su nivel de habla es regular, sin embargo esto no les supone ninguna dificultad para hablar con cualquiera; es decir, no se inhiben en su comunicación oral a pesar del reconocimiento de sus limitaciones.

En cambio, hay pacientes que se sienten bastante satisfechos con su nueva voz y sin embargo limitan su comunicación al ámbito familiar.

Estos datos nos hacen pensar en la relativa importancia de la calidad de la voz erigmofónica a la hora de realizar un acto comunicativo, pues es cierto que los pacientes, que mantiene intactos sus órganos articulatorios, son capaces de hablar sin voz y sus interlocutores de entenderles. También tendríamos que tener en cuenta aquí la importancia de la comunicación no verbal, ya que para algunos autores supone el $60 \%$ del acto comunicativo.

\section{Ansiedad y Depresión y su relación con las variables independientes}

Hemos analizado cada una de las variables independientes y las hemos relacionado con las variables dependientes ansiedad y depresión, para comprobar si existe alguna influencia de las primeras en los niveles de ansiedad medidos por el HAD (En las tablas RANS o Retest de ansiedad) y el STAI-E (en las tablas RAE o Retest de Ansiedad-Estado) y de depresión (en las tablas RDEP o Retest de Depresión medido por el HAD) mostrados por los pacientes en el momento de haber conseguido el aprendizaje de la voz erigmofónica. Hemos utilizado los estadísticos U de Mann Whitney y Prueba de KruskalWallis, con un nivel de significación de 0,05 (ver tabla 7) y también hemos realizado análisis de varianza (ANOVA), que nos permite presentar algunos gráficos en los que se muestran claramente las tendencias de las respuestas. 
Tabla 7. Significación variables independientes (retest). a: U de Mann-Witney. b: Prueba de Kruskal-Wallis. *Nivel de significación 0,05.

\begin{tabular}{|c|c|c|c|}
\cline { 2 - 4 } \multicolumn{1}{c|}{} & RANS & RA/E & RDEP \\
\hline ¿Ha aprendido a hablar? &, $135 \mathrm{a}$ &, $048 \mathrm{a}$ & $0,005 \mathrm{a}^{*}$ \\
\hline Impresión habla correcta &, $975 \mathrm{a}$ &, $166 \mathrm{a}$ & $0,248 \mathrm{a}$ \\
\hline Tiempo de aprendizaje & $0,257 \mathrm{~b}$ &, $249 \mathrm{a}$ & $0,699 \mathrm{~b}$ \\
\hline ¿Con quién habla? &, $259 \mathrm{a}$ &, $844 \mathrm{a}$ & $012 \mathrm{a}^{*}$ \\
\hline Habla con desconocidos & $3 \mathrm{a}$ &, $470 \mathrm{a}$ & $0,406 \mathrm{a}$ \\
\hline ¿Cómo habla? &, $581 \mathrm{a}$ & $021 \mathrm{a}^{*}$ \\
\hline
\end{tabular}

RANS: retest ansiedad.

$\mathrm{RA} / \mathrm{E}$ retest ansiedad /estado.

RDEP: retest depresión.

Tabla 8. ¿Ha aprendido a hablar?: U de Mann-Whitney.

\begin{tabular}{|c|r|r|r|}
\hline ¿Ha aprendido a hablar? & RDEP & \multicolumn{1}{|c|}{ RANS } & \multicolumn{1}{|c|}{ RA/E } \\
\hline U de Mann-Whitney & 14,500 & 38,500 & 29,000 \\
W de Wilcoxon & 680,500 & 704,500 & 695,000 \\
Z & $-2,628$ & $-1,520$ & $-1,974$ \\
Sig. asintót. (bilateral) &, 009 &, 129 &, 048 \\
Sig. exacta [2*(Sig. unilateral)] &, $005^{\text {a }}$ &, $135^{\text {a }}$ &, $053^{\text {a }}$ \\
\hline
\end{tabular}

RDEP: retest depresión.

RANS: retest ansiedad.

RA/E: retest ansiedad-estado.

¿Ha aprendido a hablar?

Encontramos que en los niveles de depresión, la diferencia es significativa al nivel de 0,05 entre los que han aprendido a hablar y los que no lo han hecho, a favor de los primeros $(p=0,005)$, y observamos que hay una diferencia de casi 16 puntos entre los rangos promedio $(34,88-18,90)$ (ver tabla 8). Las diferencias en los niveles de ansiedad (RANS y RAE) no son significativas. Por lo tanto, podríamos decir que el hecho de haber aprendido a hablar, puede ser una variable que influya en el nivel de depresión de los pacientes laringectomizados; no así en el de ansiedad (ver figura 1).
Figura 1. ¿Ha aprendido a hablar?

(ANOVA). Diferencias en depresión en el retest (RDEP).

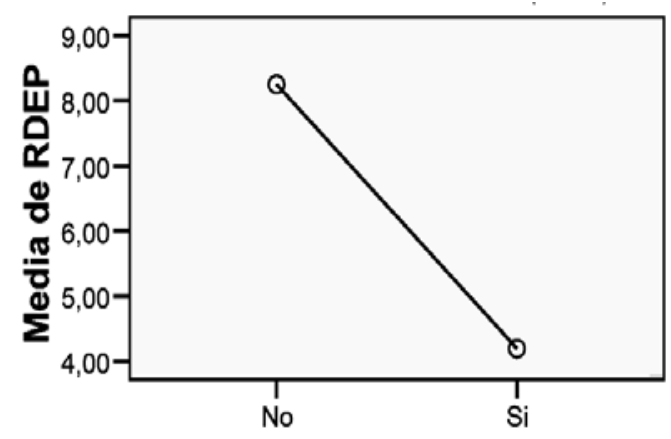

ha aprendido a hablar 
Tabla 9. Impresión habla correcta: U de Mann-Witney.

\begin{tabular}{|c|r|r|r|}
\hline Impresión habla correcta & \multicolumn{1}{|c|}{ RDEP } & \multicolumn{1}{|c|}{ RANS } & \multicolumn{1}{c|}{ RA/E } \\
\hline U de Mann-Whitney & 103,000 & 138,500 & 97,500 \\
\hline W de Wilcoxon & 148,000 & 183,500 & 142,500 \\
\hline$Z$ & $-1,199$ &,- 033 & $-1,385$ \\
\hline Sig. asintót. (bilateral) &, 231 &, 974 &, 166 \\
\hline Sig. exacta $[2 *$ Sig. unilateral)] &, $248^{\text {a }}$ &, $975^{\text {a }}$ &, $177^{\text {a }}$ \\
\hline
\end{tabular}

RDEP: retest depresión.

RANS: retest ansiedad.

RA/E: retest ansiedad-estado.

Tabla 10. Tiempo de aprendizaje (meses): Prueba Kruskal-Wallis.

\begin{tabular}{|c|c|c|c|}
\hline $\begin{array}{c}\text { Tiempo de aprendizaje } \\
\text { Meses }\end{array}$ & RDEP & RANS & RA/E \\
\hline Chi-cuadrado & 1,428 & 4,039 &, 656 \\
\hline gl & 3 & 3 & 3 \\
\hline Sig. asintót. &, 699 &, 257 &, 884 \\
\hline
\end{tabular}

RDEP: retest depresión.

RANS: retest ansiedad.

RA/E: retest ansiedad-estado.

Impresión de habla correcta

Al relacionar las medias de depresión (RDEP) y de ansiedad (RANS y RA/E) con la impresión de habla correcta, no encontramos una relación significativa $(p=0,248$, $\mathrm{p}=0,975$ y $\mathrm{p}=0,177$ respectivamente) y los rangos promedio no pasan de 5 puntos de diferencia (ver tabla 9).

Tiempo de aprendizaje de la voz erigmofónica

El tiempo que han tardado los pacientes en conseguir un nivel de habla funcional con la voz erigmofónica, medido en meses, no aparece como una variable significativa respecto a los niveles de ansiedad y de depresión (ver tabla 10).
¿Con quién habla?

A la pregunta relacionada con el valor funcional de su nueva voz adquirida, es decir, si hablan con cualquiera o solamente con sus familiares más cercanos, no se han encontrado diferencias significativas en ansiedad (RANS $p=0,259$, RAE $p=0,259$ ). Sin embargo si se dan diferencias significativas en los niveles de depresión entre los pacientes que hablan con cualquiera y los que solamente hablan con sus familiares (RDEP $\mathrm{p}=0,012$ ), a favor de los primeros; por lo que la función social del lenguaje parece tener incidencia en el nivel de depresión de los pacientes laringectomizados (ver tabla 11 y figura 2). 
Tabla 11. ¿Con quién habla?: U de Mann-Whitney

\begin{tabular}{|c|c|c|c|}
\hline ¿Con quién habla? & RDEP & RANS & RA/E \\
\hline U de Mann-Whitney & 71,500 & 113,500 & 113,500 \\
\hline W de Wilcoxon & 536,500 & 578,500 & 578,500 \\
\hline Z & $-2,486$ & $-1,147$ & $-1,153$ \\
\hline Sig. asintót. (bilateral) & ,013 & ,251 & ,249 \\
\hline $\begin{array}{l}\text { Sig. exacta }[2 *(\text { Sig. } \\
\text { unilateral })]\end{array}$ &, $012^{\mathrm{a}}$ & $259^{a}$ & ,259a \\
\hline
\end{tabular}

RDEP: retest depresión.

RANS: retest ansiedad.

RA/E: retest ansiedad-estado.

Figura 2. ¿Con quién habla? (ANOVA). Diferencias en depresión en el retest (RDEP).

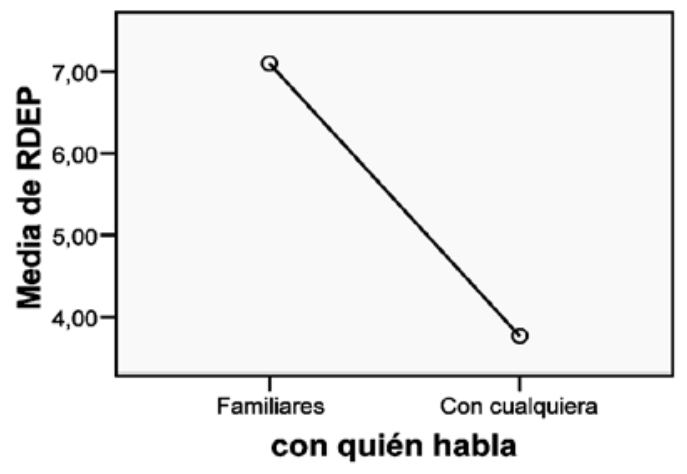

¿Cómo habla?

La consideración por parte del propio paciente de cuál es su nivel de habla, bueno o regular, es otra variable que hemos analizado en relación a los niveles de ansiedad y de depresión. En este caso comprobamos que los pacientes que consideran que solamente hablan regular presentan niveles de depresión significativamente mayores que los que consideran que hablan bien $(p=0,021)$, por lo que el nivel de habla adquirido también se ofrece como una variable a tener en cuenta al valorar el nivel de depresión de los pa- cientes laringectomizados. Los niveles de ansiedad, en cambio, no ofrecen diferencias significativas (RANS $p=0,581$; RAE $p=$ 0,500 ) (ver tabla 12 y figura 3 ).

\section{DISCUSIÓN}

Hemos analizado una serie de variables independientes referidas a la calidad de la voz erigmofónica y su funcionalidad bajo el prisma de la propia percepción de los pacientes laringectomizados y su posible relación con los niveles de ansiedad y de depresión. No hemos encontrado estudios referidos a estos aspectos, por lo que no podemos comparar nuestros resultados con los de otras investigaciones análogas. A la hora de analizar los resultados, somos conscientes de las limitaciones de nuestro estudio debido principalmente al tamaño reducido de la muestra y al ámbito concreto de ubicación del estudio, la Asociación Navarra de Laringectomizados; pero consideramos de interés la aportación de este estudio, porque tiene en cuenta la propia opinión del paciente sobre su nueva herramienta de comunicación.

Habitualmente observamos como ansiedad y depresión son dos de las consecuencias psicológicas que van unidas a los pacientes oncológicos y que se manifiestan de manera al menos parecida en 
Tabla 12. ¿Cómo habla? U de Mann-Whitney.

\begin{tabular}{|c|r|r|r|}
\hline ¿Cómo habla? & \multicolumn{1}{|c|}{ RDEP } & \multicolumn{1}{|c|}{ RANS } & \multicolumn{1}{c|}{ RA/E } \\
\hline U de Mann-Whitney & 31,000 & 71,000 & 68,000 \\
\hline W de Wilcoxon & 46,000 & 86,000 & 83,000 \\
\hline Z & $-2,303$ &,- 592 &,- 723 \\
\hline Sig. asintót. (bilateral) &, 021 &, 554 &, 470 \\
\hline Sig. exacta [2*(Sig. unilateral)] &, $021^{\mathrm{a}}$ &, $581^{\mathrm{a}}$ &, $500^{\mathrm{a}}$ \\
\hline
\end{tabular}

RDEP: retest depresión.

RANS: retest ansiedad.

RA/E: retest ansiedad-estado.

Figura 3. ¿Cómo habla? (ANOVA). Diferencias en depresión en el retest (RDEP).

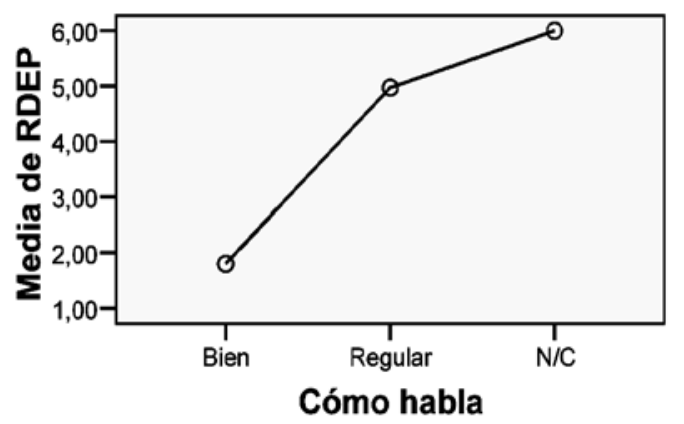

cuanto a su incidencia. En nuestro estudio no observamos tal coincidencia; al contrario, observamos que mientras los niveles de ansiedad pueden permanecer prácticamente inalterados y en unos niveles bajos, los niveles de depresión varían significativamente y nos encontramos con sujetos con niveles muy dispares en ambas; es decir, nos encontramos con pacientes con bajos niveles de ansiedad y altos niveles de depresión; todo ello con la prudencia que los límites de nuestra investigación aconsejan.

El aprendizaje de la voz erigmofónica supone un gran logro para el paciente laringectomizado, ya que le permite recuperar una herramienta fundamental en la comunicación con otras personas; pero su adquisición no es fácil y sus características, intensidad, duración, timbre, fluidez y entonación, difieren mucho de la voz laríngea que poseía el paciente, por lo que necesita un periodo de adaptación y de identificación como su propia voz. La impresión que el propio paciente tiene de su nueva voz y la funcionalidad de la misma para mantener una comunicación oral con cualquiera, se muestran como variables que pueden incidir en su estado emocional, más allá de las características físico-acústicas que pueda presentar en cada paciente.

\section{CONCLUSIÓN}

Los resultados obtenidos nos indican que existen algunas variables relativas a la voz erigmofónica que pueden influir en los niveles de depresión; no así en los niveles de ansiedad. Considera que ha aprendido a hablar, considera que habla bien y con quién habla. Estas son las variables que presentan relación con los niveles de depresión. En concreto, los pacientes que consideran que han aprendido a hablar, que hablan bien y que hablan con cualquiera, muestran niveles depresión inferiores a los que consideran que no han aprendido a hablar, consideran que solo hablan regular y que su voz les permite comunicarse solamente con familiares. 
Otras variables como el tiempo que les ha costado adquirir la voz erigmofónica, la impresión sobre la corrección de su habla o la propia satisfacción con su nueva voz, no hemos encontrado que tengan una influencia significativa con los niveles de depresión.

En cuanto a los niveles de ansiedad, no hemos encontrado ninguna de estas variables que tengan una influencia significativa en la variación de dichos niveles; aunque sí se marca una tendencia a poseer puntuaciones más altas en aqueIlos pacientes que consideran que no han aprendido a hablar, que consideran que hablan solamente regular y que hablan solamente en el ámbito reducido familiar; pero esta variación en las puntuaciones no es significativa.

Finalmente, atendiendo a los resultados obtenidos globalmente en nuestra investigación y al análisis de las diferentes variables, hemos realizado un perfil del paciente laringectomizado que no ha conseguido reducir su nivel de depresión después de haber logrado comunicarse a través de la voz erigmofónica, en el que, efectivamente, hay factores relacionados con la voz erigmofónica; pero también otros y no podemos determinar la importancia relativa de cada uno de ellos, por lo que si bien es cierta la posible influencia de las variables relativas al aprendizaje de la voz erigmofónica, éstas no deben ser consideradas como las únicas causantes de la evolución de los estados de ánimo de los pacientes laringectomizados totales.

\section{Perfil:}

- Considera que ha aprendido a hablar con dificultad, ya que le ha costado más de 6 meses; pero no se siente nada satisfecho con su nueva voz, tiene la impresión de no hablar correctamente, habla solamente con familiares y la pérdida de la voz le supuso un sentimiento de incapacidad.

- La laringectomía le afectó mucho psi- cológicamente, familiarmente, socialmente y laboralmente (está en paro).

- Se siente muy afectado físicamente, con molestias por el traqueostoma, dificultades en la respiración y dificultades para tragar alimentos.

- Es relativamente joven (54 años) y está viudo.

\section{REFERENCIAS BIBLIOGRÁFICAS}

1. González Martínez MT. Trastornos psicológicos en sujetos con cáncer de laringe: Ansiedad antes de la laringectomía. Ansiedad y depresión después de la laringectomía. Tesis Doctoral. Universidad de Salamanca, 1997.

2. Algaba J, Alduan MV, Sagastagoitia J. ¿Qué es un laringectomizado? Acta Otorrinolaringol Esp 1988; 39:21-6.

3. García Iriarte MT, O'Connor Reina C. Métodos de recuperación de la voz tras una laringectomía total. En: Cuenca Villarín MH, Barrio Parra MM, editores. El cáncer de laringe en la mujer: rehabilitación vocal, física y emocional. Madrid: Círculo Rojo, 2011.

4. Borragán A, González MJ, Agudo M. ¿Tan difícil es hablar con voz esofágica. Obstáculos habituales al aprender la voz esofágica. El cáncer de laringe en la mujer: rehabilitación vocal, física y emocional. Madrid: Círculo Rojo, 2011.

5. Zigmond AS, Snaith RP. The Hospital Anxiety and Depression Scale. Acta Psychiat. Scand; 1983; 67:361-70. Doi:10.1111/j.1600-0447.1983.tb09716.x

6. Caro I, Ibáñez E. Escala hospitalaria de ansiedad y depresión. Su utilidad práctica en Psicología de la salud. Bol Psicol 1992; 36: 43-69.

7. Spielberger CD, Gorsuch RL, Lushene RE, Cuestionario de Ansiedad Estado-Rasgo. Publicaciones de Psicología Aplicada. Madrid: TEA Eds. 5ª Ed, 1999. 